\title{
An inventory of Korean living collections in the Arnold Arboretum of Harvard University, USA
}

\author{
Sang Jun Lee, Jung-Hyun Kim, Gi-Heum Nam, Min-Ha Kim and Chae Eun Lim* \\ National Institute of Biological Resources, Incheon 404-170, Korea \\ *Correspondent: chaelim@korea.kr
}

\begin{abstract}
As part of a study to understand the current status of Korean plant species propagated in foreign countries, we conducted extensive surveys on Korean living collections in the Arnold Arboretum of Harvard University, the oldest public arboretum in North America. Specifically, we collected and identified the voucher specimens of the living collections and further examined the collection databases to understand the history of how Korean plants were introduced to the Arboretum. We found that a total of 154 Korean taxa (belonging to 49 families and 81 genera) have been planted in the arboretum. Most plants originated from seeds that were directly collected in Korea or obtained via seed exchanges with botanical gardens in Asia, Europe, and U.S.A. Of the 154 taxa, 16 are Korean endemic species, and three are listed as endangered species. In addition, 62 taxa are as floristic regional indicator species of Korea used by the Ministry of Environment.
\end{abstract}

Keywords: Arnold Arboretum, endangered species, endemic species, floristic regional indicator species, Korean living collection

(C) 2014 National Institute of Biological Resources

DOI: $10.12651 /$ JSR.2014.3.2.183

\section{INTRODUCTION}

Since the $19^{\text {th }}$ century, investigations on Korean flora were carried out to explore unrecorded flora for further systematic research and to broaden the genetic pool of known species by Japanese botanists and Western botanists including Europe and North America (Chung et al., 1986; Aiello, 2009).

In North America, the Arnold Arboretum of Harvard University is one of the oldest public arboreta and is located in the Jamaica Plain section of Boston, Massachusetts, U.S.A. $\left(42^{\circ} 19^{\prime} \mathrm{N}, 71^{\circ} 05^{\prime} \mathrm{W}\right)$. The 107 ha arboretum is a unique blend of research institution and public park, because it was founded as a public-private partnership between the City of Boston and Harvard University (President and Fellows of Harvard College, 2012). After being founded in 1872, domestic and international woody plant exploration and the subsequent evaluation of plant acquisitions have represented important missions of the Arnold Arboretum (Wyman, 1956; President and Fellows of Harvard College, 2012). Thus, the Arnold Arboretum has supported botanical exploration programs to collect germplasm in the floristically rich regions of Eastern Asia and North America since the early 1900s (Sax, 1949; Spongberg and Weaver, 1978; Howard, 1980a; 1980b; President and Fellows of Harvard College, 2012).
In particular, early arboretum staff members of the Arnold Arboretum participated in several collection expeditions to South Korea (Wilson, 1919; Sax, 1949; Spongberg, 1978; Spongberg and Weaver, 1978; Kim et al., 2010). The plant collections assimilated on these expeditions, including seeds, cuttings, scions, and budwood, were brought to the Arnold Arboretum for propagation and subsequent evaluation (Wilson, 1919; Rehder, 1930). As a result of the above activities, many plants from Korea are probably now cultivated in the Arnold Arboretum. Recently, Shim and Seo (1995a; 1995b) conducted an extensive literature review of nursery plant source lists, plant inventories, and plant exploration reports from North American arboreta, including the Arnold Arboretum. However, this study primarily focused on the commercial usage of Korean plants, depending on the existing literature reviews (Seo et al., 1996). Thus, the current list of Korean living collection is required to figure out what kinds of Korean wild plant species were brought to and cultivated in the North America.

Living plant collections, as well as herbaria, have great potential to meet future scientific needs, including taxonomy, ecology, and plant conservation (Medbury, 1991; Havens et al., 2004; Dosmann, 2006). Therefore, an inventory of living collections in arboreta is the necessary first step towards enhancing scientific research, as a 
source of raw materials, as well as towards developing a long-term resource management plan (Dosmann, 2007; Ruch et al., 2007). Since being founded in 2007, the National Institute of Biological Resources (NIBR) has obtained the collection information of Korean specimens and living materials deposited in foreign herbaria, arboreta, and museums for biological resource management (NIBR, 2008; 2009; 2010; 2011b; 2012b; 2013). As part of the above-mentioned research project, we visited the Arnold Arboretum of Harvard University, which is one of the oldest arboreta in North America.

In the present study, we collected and identified voucher specimens in the Korean living collections of the Arnold Arboretum to obtain a current inventory. Subsequently, we examined the collection database, including collector, collection date, and location of the living collections, to understand the introduction history of Korean plants to the Arnold Arboretum.

\section{Materials ANd Methods}

The species inventory of Korean plants brought to the Arnold arboretum was made by site visit during May and September 2008. With the property owner's permission, voucher specimens from the Korean living collections were collected or photographs of the Korean living collections were taken. More than 400 specimens were collected at the Arnold Arboretum. Voucher specimens were deposited in the herbarium of the NIBR (KB), South Korea. Plant identification was carried out using the available literature (Chung, 1957; Lee, 1980; 2003; Lee, 1996a; Lee, 1996b). Nomenclature and the list of identified taxa were updated and compiled according to the classification system proposed by the Genera of the Vas- cular Plants of Korea (Flora of Korea Editorial Committee, 2007).

Korean endemic species and endangered species were checked and arranged according to "Endemic Species of Korea" (NIBR, 2011a) and the "Red Data Book of Endangered Vascular Plants in Korea" (NIBR, 2012a), respectively. To understand the history of Korean plant introduction into the Arnold Arboretum, we examined collection information such as collector, collection date, and location in their databases (President and Fellows of Harvard College, 2012).

\section{Results AND Discussion}

The Korean living collections at the Arnold Arboretum consists of 154 vascular plant taxa (including 16 taxa identified to the level of variety, subspecies, or forma), representing 81 genera and 49 families (Appendix 1). Voucher specimens were designated for all Korean living collections with few exceptions, and deposited in KB (Appen$\operatorname{dix} 1)$.

In the collection, the most diverse family was the Rosaceae with 17 taxa, followed by Oleaceae (17 taxa), Betulaceae (9 taxa), Aceraceae (8 taxa), and Fabaceae (7 taxa). These 5 families represent 58 of the 154 taxa (37.6 $\%$ ) of the Korean living collections documented at the arboretum. The most diverse genus was Acer, with 8 taxa, followed by Rhododendron (6 taxa), Ligustrum (5 taxa), Malus (5 taxa), Spiraea (5 taxa), and Syringa (5 taxa). In addition, 16 taxa of eleven families were endemic to Korea (Table 1, Appendix 1). Three endangered species of Korea were propagated in the Arnold Arboretum (Table 1, Appendix 1). Cotoneaster wilsonii (Rosaceae), which is designated as "Category I" endangered

Table 1. List of Korean endemic species and endangered species planted in the Arnold Arboretum, Harvard University, U.S.A.

\begin{tabular}{|c|c|c|c|}
\hline Family & Taxa & Endemic species & Endangered species \\
\hline Pinaceae & Abies koreana E.H. Wilson 구상나무 & 0 & \\
\hline \multirow[t]{2}{*}{ Berberidaceae } & Berberis koreana Palib. 매자나무 & O & \\
\hline & Berberis amurensis var. quelpaertensis Nakai 섬매발톱나무 & O & \\
\hline Celtidaceae & Celtis choseniana Nakai 검팽나무 & 0 & \\
\hline Theaceae & Stewartia koreana Nakai ex Rehder 노각나무 & 0 & \\
\hline \multirow[t]{2}{*}{ Ericaceae } & Rhododendron aureum Georgi 노랑만병초 & & Category II \\
\hline & Rhododendron mucronulatum Turcz. 진달래 & O & \\
\hline Hydrangeaceae & Philadelphus schrenkii Rupr. 고광나무 & 0 & \\
\hline Rosaceae & Cotoneaster wilsonii Nakai 섬개야광나무 & 0 & Category I \\
\hline Fabaceae & Maackia fauriei (H. Lév.) Takeda 솔비나무 & 0 & \\
\hline \multirow[t]{5}{*}{ Oleaceae } & Forsythia koreana (Rehder) Nakai 개나리 & O & \\
\hline & Forsythia ovata Nakai 만리화 & O & \\
\hline & Fraxinus chiisanensis Nakai 물들메나무 & 0 & \\
\hline & Abeliophyllum distichum Nakai 미선나무 & 0 & Category II \\
\hline & Ligustrum foliosum Nakai 섬쥐똥나무 & O & \\
\hline Diervillaceae & Weigela subsessilis (Nakai) L.H. Bailey 병 꽃나무 & O & \\
\hline Caprifoliaceae & Lonicera subsessilis Rehder 청괴불나무 & 0 & \\
\hline
\end{tabular}


Table 2. Floristic regional indicator species listed by the Korean Ministry of Environment that were identified in the Arnold Arboretum, Harvard University, U.S.A.

\begin{tabular}{|c|c|c|c|}
\hline Category & Family & Taxa & Korean Name \\
\hline V & $\begin{array}{l}\text { Cupressaceae } \\
\text { Ericaceae } \\
\text { Rosaceae } \\
\text { Oleaceae }\end{array}$ & $\begin{array}{l}\text { Thuja koraiensis Nakai } \\
\text { Rhododendron aureum Georgi } \\
\text { Cotoneaster wilsonii Nakai } \\
\text { Abeliophyllum distichum Nakai } \\
\text { Forsythia ovata Nakai }\end{array}$ & $\begin{array}{l}\text { 눈측백 } \\
\text { 노랑만병초 } \\
\text { 섬개야광나무 } \\
\text { 미선나무 } \\
\text { 만리화 }\end{array}$ \\
\hline IV & $\begin{array}{l}\text { Pinaceae } \\
\text { Ericaceae } \\
\text { Leguminosae } \\
\text { Aceraceae } \\
\text { Oleaceae } \\
\text { Caprifoliaceae }\end{array}$ & $\begin{array}{l}\text { Tsuga sieboldii Carrière. } \\
\text { Rhododendron weyrichii Maxim. } \\
\text { Maackia fauriei } \text { (H. Lév.) Takeda } \\
\text { Acer tegmentosum Maxim. } \\
\text { Ligustrum folosum Nakai } \\
\text { Ligustrum salicinum Nakai } \\
\text { Syringa wolfi C.K. Schneid. } \\
\text { Lonicera chrysaniha Turcz. ex Ledeb. }\end{array}$ & $\begin{array}{l}\text { 솔송나무 } \\
\text { 참꽃나무 } \\
\text { 솔비나무 } \\
\text { 산겨릅나무 } \\
\text { 섬쥐똥나무 } \\
\text { 버들쥐똥나무 } \\
\text { 꽃개회나무 } \\
\text { 각시괴불나무 }\end{array}$ \\
\hline III & $\begin{array}{l}\text { Pinaceae } \\
\text { Aristolochiaceae } \\
\text { Berberidaceae } \\
\text { Ulmaceae } \\
\text { Betulaceae } \\
\text { Theaceae } \\
\text { Ericaceae } \\
\text { Rosaceae }\end{array}$ & $\begin{array}{l}\text { Abies koreana E.H. Wilson } \\
\text { Abies nephrolepis (Trautv.) Maxim. } \\
\text { Picea jezoensis (Siebold \& Zucc.) Carrière } \\
\text { Aristolochia manshuriensis Kom. } \\
\text { Berberis koreana } \text { Palib. } \\
\text { Celtis aurantiaca Nakai } \\
\text { Betula costata Trautv. } \\
\text { Betula davurica } \text { Pall. } \\
\text { Stewartia koreana Nakai ex Rehder } \\
\text { Rhododendron brachycarpum D. Don ex G. Don } \\
\text { Sorbaria sorbifolia var. stellipila Maxim. } \\
\text { Spiraea fritschiana } \text { C.K. Schneid. } \\
\text { Spiraea salicifolia } \text { L. } \\
\text { Spiraea trichocarpa } \text { Nakai } \\
\text { Ilex crenata Thunb. } \\
\text { Koelreuteria paniculata Laxm. } \\
\text { Acer barbinerve Maxim. } \\
\text { Acer palmatum Thunb. } \\
\text { Callicarpa mollis Siebold \& Zucc. } \\
\text { Ligustrum ovalifolium } \text { Hassk. } \\
\text { Syring areticulata } \text { var. mandshurica (Maxim.) H. Hara } \\
\text { Lonicera subsessilis } \text { Rehder }\end{array}$ & $\begin{array}{l}\text { 구상나무 } \\
\text { 분비나무 } \\
\text { 가문비나무 } \\
\text { 등칡 } \\
\text { 매자나무 } \\
\text { 산팽나무 } \\
\text { 거제수나무 } \\
\text { 물박달나무 } \\
\text { 노각나무 } \\
\text { 만병초 } \\
\text { 쉬땅나무 } \\
\text { 참조팝나무 } \\
\text { 꼬리조팝나무 } \\
\text { 갈기조팝나무 } \\
\text { 꽝꽝나무 } \\
\text { 모감주나무 } \\
\text { 청시닥나무 } \\
\text { 단풍나무 } \\
\text { 새비나무 } \\
\text { 왕쥐똥나무 } \\
\text { 개회나무 } \\
\text { 청괴불나무 }\end{array}$ \\
\hline II & $\begin{array}{l}\text { Berberidaceae } \\
\text { Ulmaceae } \\
\text { Aceraceae } \\
\text { Rutaceae } \\
\text { Caprifoliaceae }\end{array}$ & $\begin{array}{l}\text { Berberis amurensis Rupr. } \\
\text { Ulmus laciniata (Trautv.) Mayr } \\
\text { Acer mandshuricum Maxim. } \\
\text { Phellodendron amurense Rupr. } \\
\text { Weigela florida (Bunge) A. DC. }\end{array}$ & $\begin{array}{l}\text { 매발톱나무 } \\
\text { 난티나무 } \\
\text { 복장나무 } \\
\text { 황벽나무 } \\
\text { 붉은병꽃나무 }\end{array}$ \\
\hline I & $\begin{array}{l}\text { Pinaceae } \\
\text { Cephalotaxaceae } \\
\text { Aristolochiaceae } \\
\text { Ulmaceae } \\
\text { Fagaceae } \\
\text { Betulaceae } \\
\text { Tiliaceae } \\
\text { Rosaceae }\end{array}$ & $\begin{array}{l}\text { Abies holophylla Maxim. } \\
\text { Pinus koraiensis Siebold \& Zucc. } \\
\text { Cephalotaxus harringtonia (Knight ex Forbes) K. Koch } \\
\text { Aristolochia contorta Bunge } \\
\text { Hemiptelea davidii (Hance) Planch. } \\
\text { Ulmus parvifolia Jacq. } \\
\text { Quercus variabilis Blume } \\
\text { Betula schmidtii Regel } \\
\text { Grewia parviflora Bunge } \\
\text { Tilia amurensis Rupr. } \\
\text { Pyrus ussuriensis Maxim. } \\
\text { Rhodotypos scandens (Thunb.) Makino } \\
\text { Spiraea pubescens Turcz. } \\
\text { Wikstroemia trichotoma } \text { (Thunb.) Makino } \\
\text { Euonymus bungeanus Maxim. } \\
\text { Buxus koreana (Nakai ex Rehder) T.H. Chung, P.S. Toh, } \\
\text { D.B. Lee \& F.J. Lee } \\
\text { Eleutherococcus sessiliflorus (Rupr. \& Maxim.) S.Y. Hu } \\
\text { Chionanthus retusa Lindl. \& Paxton } \\
\text { Fraxinus mandshurica } \text { Rupr. } \\
\text { Ligustrum japonicum Thunb. } \\
\text { Syringa patula } \text { (Palib.) Nakai } \\
\text { Weigela praecox (Lemoine) L.H. Bailey }\end{array}$ & $\begin{array}{l}\text { 전나무 } \\
\text { 잣나무 } \\
\text { 개비자나무 } \\
\text { 쥐방울덩굴 } \\
\text { 시무나무 } \\
\text { 참느릅나무 } \\
\text { 굴참나무 } \\
\text { 박달나무 } \\
\text { 장구밥나무 } \\
\text { 피나무 } \\
\text { 산돌배나무 } \\
\text { 병아리꽃나무 } \\
\text { 아구장나무 } \\
\text { 산닥나무 } \\
\text { 좀참빗살나무 } \\
\text { 회양목 } \\
\text { 오갈피나무 } \\
\text { 이팝나무 } \\
\text { 들메나무 } \\
\text { 광나무 } \\
\text { 털개회나무 } \\
\text { 소영도리나무 }\end{array}$ \\
\hline
\end{tabular}


plant, is endemic to the Ulleung Island and rarely occurs in the natural habitats (NIBR, 2012a). Rhododendron aureum and Abeliophyllum distichum (Category II) are threatened in Korea by small population size and by excessive collections for its attractive flowers and fruits, respectively (NIBR, 2012a). The floristic regional indicator species were 62 taxa: 5 taxa of category V, 8 taxa of category IV, 22 taxa of category III, 5 taxa of category II, and 22 taxa of category I (Table 2).

Since the early 1900s, staff members of the Arnold Arboretum have conducted several plant explorations to Asia, including trips to Korea, China, and Japan (Rehder, 1930; 1946; Spongberg and Weaver, 1978; Howard, 1980a; 1980b; Kim et al., 2010; President and Fellows of Harvard College, 2012). Based on the collection databases of the Korean living collections, Arnold Arboretum staff participated in three collecting expeditions to South Korea between 1905 and 1977 (Appendix 1). One of the main objectives of their plant exploration is to broaden the genetic pool of known species to extend cold hardiness, increase vigor, and obtain plants with considerable ornamental value (Wilson, 1919; Wyman, 1956; Spongberg, 1993; Seo et al., 1996; Dosmann, 2007). In this regard, their collection was mainly concentrated in outstanding ornamental woody plants, including Rosaceae and Oleaceae (Appendix 1). On these expeditions, seeds and cuttings of Korean vascular plants were collected and brought to the Arnold Arboretum for propagation, the formation of hybrids, and the improvement through breeding to enhance cold resistance, flower size, and flower color (DeWolf, 1968; Dosmann, 2008). Based on the collection information, $62.5 \%$ of Korean living collections in the Arnold Arboretum were introduced from 23 Arboreta of Europe, Asia, and North America (NIBR, 2008). Thus, the history of the Korean living collection in the Arnold Arboretum includes acquisitions through seed exchange programs, as well as international plant exploration.

As the current inventory of Korean living collections in the Arnold Arboretum, these results are expected to contribute towards supporting collection-based research using documented wild sources, as well as understanding the history of Korean plant introduction in the Arnold Arboretum.

\section{ACKNOWLedgements}

The authors are grateful to the Arnold Arboretum of Harvard University for permitting this project. This project was supported by a grant entitled "An Inventory of Korean Collections deposited in Foreign Herbaria and Museums" from the National Institute of Biological Resources (NIBR) funded by the Ministry of Environment (MOE) of the Republic of Korea.

\section{RefERENCES}

Aiello, A.S. 2009. Seeking cole-hardy Camellias. Arnoldia 67:23-30.

Chung, T.H. 1957. Korean Flora II. Schinzisa, Seoul (in Korean).

Chung, Y.H., H.-K. Choi, B.-Y. Sun, Y.-C. Chung and K.-J. Kim. 1986. The history of vascular plant research. In An Introduction to the History of Plant Taxonomy in Korea. Chung, Y.H. (ed.), Academy Publishing Co., Seoul. pp. 1-190 (in Korean).

DeWolf, G.P. Jr. 1968. Albizia julibrissin and its cultivar 'Ernest Wilson'. Arnoldia. 28:28-35.

Dosmann, M.S. 2006. Research in the garden: Averting the collections crisis. Botanical Review 72:207-234.

Dosmann, M.S. 2007. The Arnold Arboretum's Living Collections: A Repository for Research. Arnoldia 65:30-39.

Dosmann, M.S. 2008. A continuing legacy: Hybrid marvels of Wilson plant introductions. Silva - The newsmagazine of the Arnold Arboretum Fall/Winter 2007-2008: 6-7.

Flora of Korea Editorial Committee. 2007. The Genera of Vascular Plants of Korea. Academy Publishing Co., Seoul.

Havens, K., E.O. Guerrant Jr., P. Vitt and M. Maunder. 2004. Conservation Research and Public Gardens. Public Gard. 19:40-43.

Howard, R.A. 1980a. E. H. Wilson as a Botanist. Arnoldia 40:102-138.

Howard, R.A. 1980b. E. H. Wilson as a Botanist. Arnoldia 40:154-193.

Kim, H., K.-S. Chang and C.-S. Chang. 2010. E. H. Wilson's Expedition to Korea from 1917 to 1919: Resolving place names of his collections. J. Jpn. Bot. 85:99-117.

Lee, T.B. 1980. Illustrated Flora of Korea. Hyangmunsa. Seoul (in Korean).

Lee, T.B. 2003. Coloured Flora of Korea $(1,2)$. Hyangmunsa. Seoul (in Korean).

Lee, W.T. 1996a. Lineamenta Florae Koreae. Academy Publisher, Seoul (in Korean).

Lee, Y.N. 1996b. Flora of Korea. Kyo-Hak Publishing Co., Seoul (in Korean).

Medbury, S. 1991. Taxonomy and garden design: a successful marriage? Public Gard. 6:29-32, 42-43.

National Institute of Biological Resources. 2008. An Inventory of Korean Collections Deposited in Foreign Herbaria and Museums (I), National Institute of Biological Resources, Seoul.

National Institute of Biological Resources. 2009. An Inventory of Korean Collections Deposited in Foreign Herbaria and Museums (II), National Institute of Biological Resources, Seoul.

National Institute of Biological Resources. 2010. An Inventory of Korean Collections Deposited in Foreign Herbaria and Museums (III), National Institute of Biological Re- 
sources, Seoul.

National Institute of Biological Resources. 2011a. Endemic Species of Korea. National Institute of Biological Resources, GeoBook Press, Seoul.

National Institute of Biological Resources. 2011b. An Inventory of Korean Collections Deposited in Foreign Herbaria and Museums (IV), National Institute of Biological Resources, Seoul.

National Institute of Biological Resources. 2012a. Red Data Book of Endangered Vascular Plants in Korea. National Institute of Biological Resources, Nature \& Ecology Press, Seoul.

National Institute of Biological Resources. 2012b. An Inventory of Korean Collections Deposited in Foreign Herbaria and Museums (V), National Institute of Biological Resources, Seoul.

National Institute of Biological Resources. 2013. An Inventory of Korean Collections Deposited in Foreign Herbaria and Museums (VI), National Institute of Biological Resources, Seoul.

President and Fellows of Harvard College. 2012. Inventory of Living Collections Compiled by the Horticulture Department. Flagship Press, North Andover, Massachusetts, USA.

Rehder, A. 1930. Ernest Henry Wilson. J. Arnold Arbor. 11: 182-192.

Rehder, A. 1946. On the history of the introduction of woody plants into North America. Arnoldia 6:13-23.

Ruch, D.G., B.G. Torke, K.S. Badger, C.R. Reidy, P.E. Rothrock, R. Waltz, E.G. Urly, J.L. Chance and L. Click.
2007. The vascular flora and vegetational communities of Hayes arboretum in Wayne county, Indiana. Proc. Indiana Acad. Sci. 116:11-41.

Sax, K. 1949. John George Jack (1861-1949) papers, 18871990. J. Arnold Arbor. 30:345-347.

Seo, B.K., Y.M. Ha and K.K. Shim. 1996. Using of Korean native plants and their cultivars in America, Canada and England. J. Kor. Flower Res. Soc. 5:49-64 (in Korean).

Shim, K.K. and B.K. Seo. 1995a. Korean native shrubs and vines in North America landscape. J. Kor. Flower Res. Soc. 4:37-62 (in Korean).

Shim, K.K. and B.K. Seo. 1995b. A Study on the Korean native woody plants of trees in the North American landscape. J. Korean Institute. Landscape Architecture 4:95-117 (in Korean).

Spongberg, S.A. 1978. Korean adventure. Arnoldia 38:133153.

Spongberg, S.A. 1993. Introducing Weigela subsessilis. Arnoldia 53:31-33.

Spongberg, S.A. and R.E. Weaver. 1978. Collecting expedition to Japan and Korea. Arnoldia 38:28-31.

Wilson, E.H. 1919. A phytogeographical sketch of the ligneous flora of Korea. J. Arnold Arbor. 1:32-43.

Wyman, D. 1956. New and rare ornamental woody plants recently distributed by the Arnold Arboretum. Arnoldia $16: 33-51$.

Submitted: June 17, 2014, Accepted: August 28, 2014 
Appendix 1. The inventory of 462 voucher specimens of Korean living collection (49 Families, 81 Genera, 153 taxa) in the Arnold arboretum is as follows. Korean endemic species are denoted with an asterisk $(*)$. In the case of the accessions without collection date, their introduction date into the Arnold Arboretum is denoted with a cross $(\dagger)$.

Family Ginkoaceae 은행나무과

1. Ginkgo biloba L. 은행나무

Examined specimens: Korea. Without specific locality: 28 Dec 1904† (NIBRVP0000283660, NIBRVP0000283661). Gyeonggi-do: Mt. Youngmun, 22 Feb 1993 (NIBRVP0000283685)

Family Pinaceae 소나무과

2. Abies holophylla Maxim. 전나무

Examined specimens: Korea. Gyeonggi-do: Gwangneung, 19 Feb 1971 † (NIBRVP0000283634); Pocheon, 22 Jun $1988 \dagger$ (NIBRVP 0000283649), 1 Jan 1987 (NIBRVP0000283635)

3. *Abies koreana E.H. Wilson 구상나무

Examined specimens: Korea. Jeju-do: Mt. Halla, 1 Jul 1917 (NIBRVP0000283647, NIBRVP0000283659), 22 May $1986 \dagger$ (NIBRVP 0000283238, NIBRVP0000283638), 22 Jun $1988 \dagger$ (NIBRVP0000283646, NIBRVP0000283671)

4. Abies nephrolepis (Trautv.) Maxim. 분비나무 Examined specimens: Korea. Without specific locality: 1 Jul 1917 (NIBRVP0000283654)

5. Picea jezoensis (Siebold \& Zucc.) Carrière 가문비나무

Examined specimens: Korea. Chungcheongnam-do: Cheollipo Arboretum, 22. Mar $1977 \dagger$ (NIBRVP0000283205, NIBRVP0000 283587)

6. Picea koraiensis Nakai 종비나무

Examined specimens: North Korea. Without specific locality: 20 Dec $1940 \dagger$ (NIBRVP0000283648), 21 Mar $1988 \dagger$ (NIBRVP0000 283651). Jagang-do: 15 Apr $1980 \dagger$ (NIBRVP0000283636)

7. Pinus densiflora Siebold \& Zucc. 소나무

Examined specimens: Korea. Without specific locality: 1 Jul 1917 (NIBRVP0000283637)

8. Pinus koraiensis Siebold \& Zucc. 잣나무

Examined specimens: Korea. Without specific locality: 1 Jul 1918 (NIBRVP0000283240), 1 Jan 1989 (NIBRVP0000283400, NIBR VP0000283650, NIBRVP0000283665). Gangwon-do: Mt. Yongmun, 24. Oct 1983† (NIBRVP0000283239, NIBRVP0000283241)

9. Tsuga sieboldii Carrière 솔송나무

Examined specimens: Korea. Gyeongsangbuk-do: Is. Ulleung, 24 Oct 1983† (NIBRVP0000283521, NIBRVP0000283579)

Family Cupressaceae 측백나무과

10. Thuja koraiensis Nakai 눈측백

Examined specimens: Korea. Gangwon-do: Mt. Taebaek, 2 Oct 1989 (NIBRVP0000283206), Mt. Seorak, 29 Mar $1997 \dagger$ (NIBRVP 0000283207)

Family Cephalotaxaceae 개비자나무과

11. Cephalotaxus harringtonia (Knight ex Forbes) K. Koch 개비자나무

Examined specimens: Korea. Jeollanam-do: Chunchon, 12 Oct 1977 (NIBRVP0000283237)

Family Magnoliaceae 목련과

12. Magnolia sieboldii K. Koch 함박꽃나무

Examined specimens: Korea. Gangwon-do: Chuncheon, Cheongpyeongsa Temple, 25 Jun $1987 \dagger$ (NIBRVP0000283339)

Family Lauraceae 녹나무과

13. Lindera erythrocarpa Makino 비목나무

Examined specimens: Korea. Jeollabuk-do: Mt. Naejang, 11 Jun $1984 \dagger$ (NIBRVP0000283489, NIBRVP0000283490). Jeollanamdo: Mt. Mudeung, 1 Oct 1978 (NIBRVP0000283330); 11 Jun $1984 \dagger$ (NIBRVP0000283500)

14. Lindera glauca (Siebold \& Zucc.) Blume 감태나무

Examined specimens: Korea. Gyeonggi-do: Seoul, Forest Research Institute, 5 Oct 1977 (NIBRVP0000283480, NIBRVP0000283481)

15. Lindera obtusiloba Blume 생강나무

Examined specimens: Korea. Gangwon-do: Mt. Seorak, 12 Aug 1982 † (NIBRVP0000283675, NIBRVP0000283676); 24 Oct 1983† (NIBRVP0000283564, NIBRVP0000283565, NIBRVP0000283664). Gyeonggi-do: Yonhwa-ri, 2 Apr 1986† (NIBRVP0000283674). Jeollabuk-do: Mt. Daedun, 1 Jul $1985 \dagger$ (NIBRVP0000283562, NIBRVP0000283563, NIBRVP0000283657, NIBRVP0000283658)

Family Aristolochiaceae 쥐방울덩굴과

16. Aristolochia contorta Bunge 쥐방울덩굴

Examined specimens: Korea. Jeju-do: Mt. Halla, 1 Jan 2000 (NIBRVP0000283309)

17. Aristolochia manshuriensis Kom. 등칡

Examined specimens: Korea. Gyeongsangbuk-do: Mt. Juwang, 22 May $1987 \dagger$ (NIBRVP0000283251, NIBRVP0000283252, NIBRVP 0000283253)

Family Schisandraceae 오미자과

18. Schisandra chinensis (Turcz.) Baill. 오미자

Examined specimens: Korea. Gangwon-do: Mt. Chiak, 8 May 1989† (NIBRVP0000283311)

Family Ranunculaceae 미나리아재비과

19. Clematis terniflora $\mathrm{DC}$. 참으아리

Examined specimens: Korea. Gyeonggi-do: Mt. Yongmun, 9 Oct 1977 (NIBRVP0000283314, NIBRVP0000283315)

Family Berberidaceae 매자나무과

20. Berberis amurensis Rupr. 매발톱나무

Examined specimens: Korea. Gyeongsangbuk-do: Is. Ulleung, 25 Jun $1987 \dagger$ (NIBRVP0000283188, NIBRVP0000283189, NIBRVP 0000283333, NIBRVP0000283334)

21. *Berberis amurensis var. quelpaertensis Nakai 섬매발톱나무

Examined specimens: Korea. Gyeonggi-do: Seoul, Forest Research Institute, 5 Oct 1977 (NIBRVP0000283219, NIBRVP0000283260, NIBRVP0000283261, NIBRVP0000283262,). Jeju-do: Mt. Halla, Yeongsil, 24 Oct $1983 \dagger$ (NIBRVP0000283263) 
Appendix 1. Continued.

22. *Berberis koreana Palib. 매자나무

Examined specimens: Korea. Without specific locality: 1 Jan 1904 (NIBRVP0000283187); 5 Aug $1983 \dagger$ (NIBRVP0000283186, NIBRVP0000283259)

23. Berberis poiretii C. K. Schneid. 당매자나무

Examined specimens: Korea. Chungcheongnam-do: Cheollipo Arboretum, 2 Oct 1977 (NIBRVP0000283342)

Family Eucommiaceae 두충과

24. Eucommia ulmoides Oliv. 두충

Examined specimens: Korea. Gyeonggi-do: Seoul, Mt. Gwanak, 2 Jun 1989† (NIBRVP0000283268)

Family Ulmaceae 느릅나무과

25. Hemiptelea davidii (Hance) Planch. 시무나무

Examined specimens: Korea. Without specific locality: 1 Dec 1904 † (NIBRVP0000283328, NIBRVP0000283329, NIBRVP0000 283470), 30 Jul $1992 \dagger$ (NIBRVP0000283245, NIBRVP0000283246)

26. Ulmus davidiana var. japonica (Rehder) Nakai 느릅나무

Examined specimens: Korea. Gangwon-do: Mt. Gyebang, 31 May 1978 (NIBRVP0000283513, NIBRVP0000283514)

27. Ulmus laciniata (Trautv.) Mayr 난티나무

Examined specimens: Korea. Gangwon-do: Mt. Odae, 14 Jun $2001 \dagger$ (NIBRVP0000283516)

28. Ulmus parvifolia Jacq. 참느릅나무

Examined specimens: Korea. Chungcheongnam-do: Seosan, 4 Apr 1986 † (NIBRVP0000283231). Gyeongsangbuk-do: Daejeon, 19 Dec $1973 \dagger$ (NIBRVP0000283202). Jeollanam-do: Suncheon, 12 Oct 1977 (NIBRVP0000283232)

29. Zelkova serrata (Thunb.) Makino 느티나무

Examined specimens: Korea. Gyeonggi-do: Mt. Yongmun, 9 Oct 1977 (NIBRVP0000283497, NIBRVP0000283498, NIBRVP0000 283499, NIBRVP0000283501, NIBRVP0000283502, NIBRVP0000283503, NIBRVP0000283504)

Family Celtidaceae 팽나무과

30. Celtis aurantiaca Nakai 산팽나무

Examined specimens: Korea. Jeollanam-do: Seonamsa Temple, 12 Oct 1977 (NIBRVP0000283509)

31. * Celtis choseniana Nakai 검팽나무

Examined specimens: Korea. Gyeonggi-do: Incheon, Is. Daecheong, 12 Oct 1984 (NIBRVP0000283200); Is. Socheong, 2 Apr 1986† (NIBRVP0000283198, NIBRVP0000283199)

Family Moraceae 뽕나무과

32. Broussonetia kazinoki Siebold 닥나무

Examined specimens: Korea. Gyeonggi-do: Incheon, Is. Daecheong, 12 Oct 1984 (NIBRVP0000283471, NIBRVP0000283472)

Family Fagaceae 참나무과

33. Quercus dentata Thunb. 떡갈나무

Examined specimens: Korea. Chungcheongnam-do: Cheollipo Arboretum, 2 Oct 1977 (NIBRVP0000283543)

34. Quercus variabilis Blume 굴참나무

Examined specimens: Korea. Chungcheongnam-do: Uihang-ri, 2 Oct 1977 (NIBRVP0000283360)

Family Betulaceae 자작나무과

35. Alnus x mayrii Callier ex C.k. Schneid. 잔털오리나무

Examined specimens: Korea. Gyeonggi-do: Incheon, Is. Daeyeonpyeong, 2 Apr $1986 \dagger$ (NIBRVP0000283510)

36. Betula costata Trautv. 거제수나무

Examined specimens: Korea. Gangwon-do: Mt. Taebaek, 1 May 1979† (NIBRVP0000283628, NIBRVP0000283629)

37. Betula dahurica Pall. 물박달나무

Examined specimens: Korea. Gangwon-do: Mt. Seorak, 16 Apr $1968 \dagger$ (NIBRVP0000283626); between Hongcheon and Chuncheon, 5 Oct 1977 (NIBRVP0000283505). Gyeonggi-do: Gwangneung, 15 Mar 1983† (NIBRVP0000283655, NIBRVP0000283656); Seoul, Hongneung Arboretum, 5 Oct 1977 (NIBRVP0000283518)

38. Betula ermanii Cham. 사스래나무

Examined specimens: Korea. Gangwon-do: Mt. Taebaek, 15 May 1979† (NIBRVP0000283653); 15 Oct 1978 (NIBRVP0000283600); 1 Oct 1978 (NIBRVP0000283517)

39. Betula schmidtii Regel 박달나무

Examined specimens: Korea. Gangwon-do: Wonju, Mt. Chiak, 14 Jun $2001 \dagger$ (NIBRVP0000283492, NIBRVP0000283493, NIBRVP 0000283494)

40. Carpinus laxiflora (Siebold \& Zucc.) Blume 서어나무

Examined specimens: Korea. Gyeonggi-do: Incheon, Ganghwa-gun, Hwado-myeon, 6 Oct 1984 (NIBRVP0000283589, NIBRVP000 0283593); Gwangneung, 15 Mar 1983 † (NIBRVP0000283588). Jeollanam-do: Mt. Jiri, 1 Jul 1985 † (NIBRVP0000283591, NIBRVP 0000283592).

41. Carpinus tschonoskii Maxim. 개서어나무

Examined specimens: Korea. Jeollanam-do: Mt. Chogye, near Sonamsa-temple, 24 Oct $1983 \dagger$ (NIBRVP0000283520)

42. Carpinus turczaninowii Hance 소사나무

Examined specimens: Korea. Chungcheongnam-do: Cheollipo Arboretum, 4 Oct 1977 (NIBRVP0000283594). Gyeonggi-do: Incheon, Ganghwa-gun, Hwado-myeon, 6 Oct 1984 (NIBRVP0000283590)

43. Corylus sieboldiana Blume 참개암나무

Examined specimens: Korea. Gangwon-do: Mt. Seorak Daecheong Peak, 22 Mar 1977† (NIBRVP0000283596, NIBRVP0000283597)

Family Theaceae 차나무과

44. *Stewartia koreana Nakai ex Rehder 노각나무

Examined specimens: Korea. Gyeongsangnom-do: Mt. Jiri, 17 Nov 1917 (NIBRVP0000283215, NIBRVP0000283216, NIBRVP00 00283554). Jeollanam-do: Mt. Mudeung, 24 Oct $1983 \dagger$ (NIBRVP0000283217) 
Appendix 1. Continued.

Family Actinidiaceae 다래나무과

45. Actinidia arguta (Siebold \& Zucc.) Planch. ex Miq. 다래

Examined specimens: Korea. Gyeongsangnam-do: Mt. Gaya, 1 Jul 1985 † (NIBRVP0000283312)

46. Actinidia polygama (Siebold \& Zucc.) Maxim. 개다래

Examined specimens: Korea. Gyeonggi-do: Gwangneung, 15 Mar 1983† (NIBRVP0000283254, NIBRVP0000283255, NIBRVP0

000283256, NIBRVP0000283257, NIBRVP0000283258). Gyeongsangbuk-do: Is. Ulleung, 24 Oct 1983 † (NIBRVP0000283308)

Family Tiliaceae 피나무과

47. Grewia parviflora Bunge 장구밥나무

Examined specimens: Korea. Chungcheongnam-do: Cheollipo Arboretum, 1 Jul $1985 \dagger$ (NIBRVP0000283190, NIBRVP0000283269). Gyeonggi-do: Incheon, Is. Baengnyeong, 2 Apr 1986† (NIBRVP0000283267); Is. Daecheong, 2 Apr $1986 \dagger$ (NIBRVP0000283270, NIBRVP0000283274)

48. Tilia amurensis Rupr. 피나무

Examined specimens: Korea. Chungcheongnam-do: Cheollipo Arboretum, 3 Oct 1976(NIBRVP0000283278)

49. Tilia koreana Nakai 연밥피나무

Examined specimens: Korea. Jeollanam-do: Mt. Jiri, 4 Jun $1990 \dagger$ (NIBRVP0000283247, NIBRVP0000283248)

Family Malvaceae 아욱과

50. Hibiscus syriacus L. 무궁화

Examined specimens: Korea. Chungcheongbuk-do: 27 Jul 1981† (NIBRVP0000283461, NIBRVP0000283630); 9 Mar 1953† (NIBRVP0000283631)

Family Ericaceae 진달래과

51. Rhododendron aureum Georgi 노랑만병초

Examined specimens: Korea. Gangwon-do: Mt. Seorak, 17 Apr 1969† (NIBRVP0000283331)

52. Rhododendron brachycarpum D. Don ex G. Don 만병초

Examined specimens: Korea. Gangwon-do: Mt. Odae, 22 Sep 1989 (NIBRVP0000283582, NIBRVP0000283583, NIBRVP0000283 584). Gyeongsangbuk-do: Is. Ulleung, 21 Oct 1989 (NIBRVP0000283575, NIBRVP0000283576, NIBRVP0000283577, NIBRVP 0000283578).

53. *Rhododendron mucronulatum Turcz. 진달래

Examined specimens: Korea. Chungcheongnam-do: Seosan, 8 Jul 1985 † (NIBRVP0000283340). Gyeonggi-do: Seoul, Mt. Gwanak, 5 Jan $1972 \dagger$ (NIBRVP0000283204), 11 Aug $2000 \dagger$ (NIBRVP0000283341, NIBRVP0000283571)

54. Rhododendron schlippenbachii Maxim. 철쭉

Examined specimens: Korea. Without specific locality: 1 Jan 1918 (NIBRVP0000283581). Gyeonggi-do: Seoul, 15 Dec $1905 \dagger$ (NIBRVP0000283580),

55. Rhododendron weyrichii Maxim. 참꽃나무

Examined specimens: Korea. Without specific locality: No collection date (NIBRVP0000283572)

56. Rhododendron yedoense f. poukhanense (H. Lév.) M. Sugim. 산철쭉

Examined specimens: Korea. Jeollanam-do: Mt. Wolchul, 11 Jun $1984 \dagger$ (NIBRVP0000283208, NIBRVP0000283585)

Family Styracaceae 때죽나무과

57. Styrax japonicus Siebold \& Zucc. 때죽나무

Examined specimens: Korea. Gangwon-do: Mt. Seorak, 7 Oct 1977 (NIBRVP0000283633)

58. Styrax obassia Siebold \& Zucc. 쪽동백나무

Examined specimens: Korea. Gangwon-do: Mt. Seorak, 17. May. 1977† (NIBRVP0000283598). Gyeonggi-do: Incheon, Ganghwagun, 2 Apr $1986 \dagger$ (NIBRVP0000283682, NIBRVP0000283683). Gyeongsangbuk-do: Mt. Toham, 24. Oct. 1983† (NIBRVP0000 283669).

Family Symplocaceae 노린재나무과

59. Symplocos sawafutagi Nagam. 노린재나무

Examined specimens: Korea. Gyeonggi-do: Gwangneung, 15 Mar 1983† (NIBRVP0000283672)

60. Symplocos tanakana Nakai 검노린재

Examined specimens: Korea. Without specific locality: 9 Dec $1968 \dagger$ (NIBRVP0000283525). Jeollanam-do: Mt. Wolchul, 11 Jun $1984 \dagger$ (NIBRVP0000283495, NIBRVP0000283496)

Family Hydrangeaceae 수국과

61. *Philadelphus schrenkii Rupr. 고광나무

Examined specimens: Korea. Without specific locality: No collection date (NIBRVP0000283454). Gangwon-do: Mt. Seorak, 4 Nov 1969 (NIBRVP0000283224, NIBRVP0000283407)

62. Philadelphus schrenckii var. jackii Koehne 털고광나무

Examined specimens: Korea. Gangwon-do: Mt. Gyebang, 8 Oct 1977 (NIBRVP0000283443, NIBRVP0000283444, NIBRVP0000 283445). Gyeonggi-do: Seoul, Mt. Bukhan, 20 Jul $1992 \dagger$ (NIBRVP0000283465, NIBRVP0000283466)

63. Philadelphus tenuifolius Rupr. ex Maxim. 엷은잎고광나무

Examined specimens: Korea. Jeju-do: Mt. Halla, 1 Jan 1987 (NIBRVP0000283430, NIBRVP0000283431, NIBRVP0000283432). Jeollanam-do: Mt. Mudeung, 22 Jun $1988 \dagger$ (NIBRVP0000283433, NIBRVP0000283434, NIBRVP0000283435)

Family Grossulariaceae 까치밥나무과

64. Ribes fasciculatum var. chinense Maxim. 까마귀밥나무

Examined specimens: Korea. Chungcheongnam-do: Cheollipo Arboretum, 2 Oct 1977 (NIBRVP0000283422). Jellanam-do: Mt. Mudeung, 22 Jun $1988 \dagger$ (NIBRVP0000283437)

65. Ribes triste Pall. 눈까치밥나무

Examined specimens: Korea. Gangwon-do: Mt. Seorak, 15 Jun $1994 \dagger$ (NIBRVP0000283438)

Family Rosaceae 장미과

66. Aria alnifolia (Siebold \& Zucc.) Decne. 팥배나무

Examined specimens: Korea. Gyeonggi-do: Incheon, Is. Socheong, 4 Apr $1986 \dagger$ (NIBRVP0000283519) 
Appendix 1. Continued.

67. *Cotoneaster wilsonii Nakai 섬개야광나무

Examined specimens: Korea. Gyeongsangbuk-do: Is. Ulleung, 25 Jun $1987 \dagger$ (NIBRVP0000283221, NIBRVP0000283387, NIBRVP 0000283388)

68. Malus asiatica Nakai 능금나무

Examined specimens: Korea. Without specific locality: 1 Jul 1918 (NIBRVP0000283569, NIBRVP0000283644)

69. Malus baccata (L.) Borkh. 야광나무

Examined specimens: Korea. Jeollanam-do: Mt. Wolchul, 11 Jun 1984 † (NIBRVP0000283697). Gyeonggi-do: Seoul, 20 Aug $1953 \dagger$ (NIBRVP0000283698); 18 Mar $1987 \dagger$ (NIBRVP0000283702, NIBRVP0000283704)

70. Malus baccata var. mandshurica (Maxim.) C. K. Schneid. 털야광나무

Examined specimens: Korea. Jeollabuk-do: Jeonju, Korea Expressway Corporation Arboretum, 11 Jun $1984 \dagger$ (NIBRVP0000283452)

71. Malus micromalus Makino 개아그배나무

Examined specimens: Korea. Jeju-do: Mt. Halla, 24 Oct $1983 \dagger$ (NIBRVP0000283693)

72. Malus sieboldii (Regel) Rehder 아그배나무

Examined specimens: Korea. Jeju-do: Mt. Halla, 22 Jun $1988 \dagger$ (NIBRVP0000283382, NIBRVP0000283383)

73. Pourthiaea villosa (Thunb.) Decne. 윤노리나무

Examined specimens: Korea. Jeollabuk-do: Buan-gun, Jinseo-myeon, 3 Oct 1985 (NIBRVP0000283368, NIBRVP0000283369, NIBRVP0000283370). Jeollanam-do: Mt. Wolchul, 11 Jun $1984 \dagger$ (NIBRVP0000283376); Mt. Jiri, 1 Jul $1985 \dagger$ (NIBRVP000028 3377, NIBRVP0000283378, NIBRVP0000283379); Wando-gun Gunoe-myeon, 21 Oct 1985 (NIBRVP0000283371, NIBRVP0000 283372)

74. Pyrus ussuriensis Maxim. 산돌배나무

Examined specimens: Korea. Jeollanam-do: Mt. Wolchul, 11 Jun $1984 \dagger$ (NIBRVP0000283540, NIBRVP0000283541)

75. Rhodotypos scandens (Thunb.) Makino 병아리꽃나무

Examined specimens: Korea. Is. So-chona: 1 Jul 1982 (NIBRVP0000283389)

76. Rosa rugosa Thunb. 해당화

Examined specimens: Korea. Gyeonggi-do: Incheon, Is. Baengnyeong, 17 Oct 1984 (NIBRVP0000283385). Pyeongannam-do: Pyeongyang, Yellow Sea shore, 6 May 1985 † (NIBRVP0000283386)

77. Sorbaria sorbifolia var. stellipila Maxim. 쉬땅나무

Examined specimens: Korea. Gangwon-do: Mt. Seorak, 15 Mar $1983 \dagger$ (NIBRVP0000283361); Mt. Odae, 15 Mar $1983 \dagger$ (NIBRVP 0000283363). Yanggang-do: Gaema Plateau and Mt. Baekdu, 6 May $1985 \dagger$ (NIBRVP0000283364)

78. Spiraea fritschiana C.K. Schneid. 참조팝나무

Examined specimens: Korea. Without specific locality: 12 Sep 1977† (NIBRVP0000283381). Gangwon-do: Mt. Seorak, 15 Mar $1983+($ NIBRVP0000283362)

79. Spiraea prunifolia var. simpliciflora (Nakai) Nakai 조팝나무

Examined specimens: Korea. Without specific locality: 1 Jan 1905 (NIBRVP0000283307); 19 Jul 1999† (NIBRVP0000283428); 14. Jun. $2002 \dagger$ (NIBRVP0000283249, NIBRVP0000283250)

80. Spiraea pubescens Turcz. 아구장나무

Examined specimens: Korea. Gangwon-do: Mt. Seorak, 15 Mar 1983 † (NIBRVP0000283302); 16 Jun $1999 \dagger$ (NIBRVP0000283303, NIBRVP0000283304, NIBRVP0000283367)

81. Spiraea salicifolia $\mathrm{L}$. 꼬리조팝나무

Examined specimens: Korea. Yanggang-do: Gaema Plateau and Mt. Baekdu, 6 May $1985 \dagger$ (NIBRVP0000283375)

82. Spiraea trichocarpa Nakai 갈기조팝나무

Examined specimens: Korea. Yanggang-do: Gaema Plateau and Mt. Baekdu, 6 May $1985 \dagger$ (NIBRVP0000283365, NIBRVP0000 283366)

Family Fabaceae 콩과

83. Albizia julibrissin Durazz. 자귀나무

Examined specimens: Korea. Without specific locality: 1 Jul 1919 (NIBRVP0000283408, NIBRVP0000283570). Gyeonggi-do: Mt. Yongmun, 9 Oct 1977 (NIBRVP0000283338)

84. Gleditsia japonica Miq. 주엽나무

Examined specimens: Korea. Chungcheongnam-do: 4 Oct 1977 (NIBRVP0000283236, NIBRVP0000283678); Cheollipo Arboretum, 1 Jan 1995 (NIBRVP0000283680); Taean, 24 Jun $1996 \dagger$ (NIBRVP0000283235, NIBRVP0000283679)

85. Indigofera kirilowii Maxim. ex Palib. 땅비싸리

Examined specimens: Korea. Gyeonggi-do: near Seoul, 13 Jul $2004 \dagger$ (NIBRVP0000283300, NIBRVP0000283301)

86. Lespedeza maximowiczii C.K. Schneid. 조록싸리

Examined specimens: Korea. Without specific locality: 1 Jul 1917 (NIBRVP0000283399)

87. Maackia amurensis Rupr. \& Maxim. 다릅나무

Examined specimens: Korea. Gangwon-do: 17 Apr $1978 \dagger$ (NIBRVP0000283524, NIBRVP0000283686, NIBRVP0000283687)

88. *Maackia fauriei (H. Lév.) Takeda 솔비나무

Examined specimens: Korea. Jeju-do: Mt. Halla, 9 Jul $1987 \dagger$ (NIBRVP0000283220)

89. Sophora japonica L. 회화나무

Examined specimens: Korea. Without specific locality: 4 Jun $1920 \dagger$ (NIBRVP0000283410). Jeollanam-do: Haenam, 24 Oct 1983 † (NIBRVP0000283568, NIBRVP0000283668)

Family Elaeagnaceae 보리수나무과

90. Elaeagnus umbellata Thunb. 보리수나무

Examined specimens: Korea. Without specific locality: 28 Dec $1904 \dagger$ (NIBRVP0000283242); 3 Aug $1990 \dagger$ (NIBRVP0000283601)

Family Thymelaeaceae 팥꽃나무과

91. Wikstroemia trichotoma (Thunb.) Makino 산닥나무

Examined specimens: Korea. Jeollabuk-do: Jeonju, Korea Expressway Corporation Arboretum, 11 Oct 1977 (NIBRVP0000283295, NIBRVP0000283296) 
Appendix 1. Continued.

Family Cornaceae 층층나무과

92. Cornus kousa F. Buerger ex Miq. 산딸나무

Examined specimens: Korea. Jeollabuk-do: Mt. Deogyu, 9 Dec $1968 \dagger$ (NIBRVP0000283401). Gyeonggi-do: Incheon, Ganghwa, 2 Apr $1986 \dagger($ NIBRVP0000283696)

93. Cornus macrophylla Wall. 곰의말채나무

Examined specimens: Korea. Jeju-do: 16 Jun $1994 \dagger$ (NIBRVP0000283264, NIBRVP0000283265); Mt. Halla, 1 Oct 1993 (NIBRVP 0000283266, NIBRVP0000283530)

94. Cornus officinalis Siebold \& Zucc. 산수유

Examined specimens: Korea. Gyeonggi-do: Seoul, Namdaemun Market, 24 Oct $1984 \dagger$ (NIBRVP0000283293, NIBRVP0000283526, NIBRVP0000283527, NIBRVP0000283528). Gyeongsangnam-do: Mt. Gaya, 1 Jul $1985 \dagger$ (NIBRVP0000283529)

95. Cornus walteri Wangerin 말채나무

Examined specimens: Korea. Chungcheongnam-do: Cheollipo Arboretum, 2 Oct 1977 (NIBRVP0000283423)

Family Celastraceae 노박덩굴과

96. Euonymus alatus f. apterus (Regel) Rehder. 당회잎나무

Examined specimens: Korea. Pyeongannam-do: Pyeongyang, 1 Dec $1905 \dagger$ (NIBRVP0000283625)

97. Euonymus bungeanus Maxim. 좀참빗살나무

Examined specimens: Korea. Jeollanam-do: Mt. Jiri, 1 Jul $1985 \dagger$ (NIBRVP0000283632)

98. Euonymus hamiltonianus Wall. 참빗살나무

Examined specimens: Korea. Chungcheongnam-do: Cheollipo Arboretum, 2 Nov 1984 (NIBRVP0000283695). Jeju-do: Mt. Halla, 24 Oct $1983 \dagger($ NIBRVP0000283688)

99. Tripterygium regelii Sprague \& Takeda 미역줄나무

Examined specimens: Korea. Gangwon-do: 6 Oct 1977 (NIBRVP0000283294, NIBRVP0000283357, NIBRVP0000283384). Gyeonggido: Seoul, Mt. Bukhan, 30 Dec $1905 \dagger$ (NIBRVP0000283624); 15 Jun 1995 † (NIBRVP0000283623, NIBRVP0000283627)

Family Aquifoliaceae 감탕나무과

100. Ilex crenata Thunb. 꽝꽝나무

Examined specimens: Korea. Jeju-do: Mt. Halla, 29 Nov $1988 \dagger$ (NIBRVP0000283618, NIBRVP0000283619, NIBRVP0000283620)

Family Buxaceae 회양목과

101. Buxus koreana (Nakai ex Rehder) T.H. Chung, P.S. Toh, D.B. Lee \& F.J. Lee 회양목

Examined specimens: Korea. Without specific locality: 1 Jul 1919 (NIBRVP0000283218)

Family Euphorbiaceae 대극과

102. Securinega suffruticosa (Pall.) Rehder 광대싸리

Examined specimens: Korea. Gangwon-do: Mt. Seorak, 7 Oct 1977 (NIBRVP0000283535, NIBRVP0000283536, NIBRVP000028 3537, NIBRVP0000283538, NIBRVP0000283539, NIBRVP0000283621, NIBRVP0000283622). Pyeongannam-do: Pyeongyang, 22. Jun. $1999 \dagger$ (NIBRVP0000283533, NIBRVP0000283534)

Family Rhamnaceae 갈매나무과

103. Hovenia dulcis Thunb. 헛개나무

Examined specimens: Korea. Gangwon-do: Mt. Seorak, 17 Oct 1977 (NIBRVP0000283652)

Family Sapindaceae 무환자나무과

104. Koelreuteria paniculata Laxm. 모감주나무

Examined specimens: Korea. Academy of Forest Sciences, North Korea, 1 Jul 2001 (NIBRVP0000283352, NIBRVP0000283353). Chungcheongnam-do: Taean, 2 Oct 1977 (NIBRVP0000283316, NIBRVP0000283317, NIBRVP0000283351)

Family Aceraceae 단풍나무과

105. Acer barbinerve Maxim. 청시닥나무 Examined specimens: Korea. Gangwon-do: Mt. Seorak, Daecheong Peak, 22 Mar $1977 \dagger$ (NIBRVP0000283354)

106. Acer buergerianum Miq. 중국단풍

Examined specimens: Korea. Jeollanam-do: Forest Research station, 18 Oct 1977 (NIBRVP0000283406, NIBRVP0000283560)

107. Acer mandshuricum Maxim. 복장나무

Examined specimens: Korea. Gangwon-do: Mt. Odae, 8 May $1989 \dagger$ (NIBRVP0000283403)

108. Acer palmatum Thunb. 단풍나무

Examined specimens: Korea. Jeollabuk-do: Mt. Naejang, 22 Jun $1988 \dagger$ (NIBRVP0000283359, NIBRVP0000283395, NIBRVP0000 283396)

109. Acer pseudosieboldianum (Pax) Kom. 당단풍나무

Examined specimens: Korea. Without specific locality: 1 Jan 1919 (NIBRVP0000283213). Gangwon-do: Mt. Seorak, 7 Oct 1977 (NIBRVP0000283356, NIBRVP0000283391, NIBRVP0000283392). Gyeonggi-do: Gwangneung, 21 Feb $1995 \dagger$ (NIBRVP000028 3192). Gyeongsangbuk-do: Is. Ulleung, 24 Oct 1983 (NIBRVP0000283214, NIBRVP0000283355); 25 Jun 1987 (NIBRVP00002 83193, NIBRVP0000283211). Jeollabuk-do: Mt. Naejang, 22 Jun $1988 \dagger$ (NIBRVP0000283212)

110. Acer tataricum ssp. ginnala (Maxim.) Wesm. 신나무 Examined specimens: Korea. Jeollanam-do: Mt. Jiri, 1 Jul $1985 \dagger$ (NIBRVP0000283402)

111. Acer tegmentosum Maxim. 산겨릅나무 Examined specimens: Korea. Gangwon-do: Mt. Gyebang, 8 Oct 1977 (NIBRVP0000283398); Mt. Odae, 22 Mar $1977 \dagger$ (NIBRVP 0000283532)

112. Acer triflorum Kom. 복자기

Examined specimens: Korea. Gangwon-do: Mt. Seorak, 13 Aug 1982 † (NIBRVP0000283463, NIBRVP0000283556). Gyeonggi-do: Gwangneung, 16 Dec $1987 \dagger$ (NIBRVP0000283358, NIBRVP0000283404)

Family Anacardiaceae 옻나무과

113. Rhus javanica $\mathrm{L}$. 붉나무

Examined specimens: Korea. Gyeonggi-do: Incheon, Is. Baengnyeong, 4 Apr $1986 \dagger$ (NIBRVP0000283703) 
Appendix 1. Continued.

Family Simaroubaceae 소태나무과

114. Picrasma quassioides (D. Don) Benn. 소태나무

Examined specimens: Korea. Gyeonggi-do: Seoul, 1 Jan 1947 (NIBRVP0000283346, NIBRVP0000283347, NIBRVP0000283348, NIBRVP0000283349, NIBRVP0000283350)

Family Rutaceae 운향과

115. Euodia daniellii Hemsl. 쉬나무

Examined specimens: Korea. Gyeonggi-do: Incheon, Ganghwa, 4 Jun $1990 \dagger$ (NIBRVP0000283344, NIBRVP0000283345)

116. Phellodendron amurense Rupr. 황벽나무

Examined specimens: Korea. Gangwon-do: Mt. Gyebang, 1 Jan 1979† (NIBRVP0000283291). Gyeongsangbuk-do: Is. Ulleung, 25 Jun $1987 \dagger$ (NIBRVP0000283292)

117. Zanthoxylum simulans Hance

Examined specimens: Korea. Gyeonggi-do: Seoul, Korea Forest Research Institute, 5 Oct 1977 (NIBRVP0000283343)

Family Araliaceae 두릅나무과

118. Aralia elata (Miq.) Seem. 두릅나무

Examined specimens: Korea. Jeollabuk-do: Mt. Naejang, 22 Jun $1988 \dagger$ (NIBRVP0000283424, NIBRVP0000283425)

119. Eleutherococcus sessiliflorus (Rupr. \& Maxim.) S. Y. Hu 오갈피나무

Examined specimens: Korea. Gangwon-do: Mt. Gyebang, 8 Oct 1977 (NIBRVP0000283233, NIBRVP0000283234). Jeollabuk-do: Iksan, 18 May $1980 \dagger($ NIBRVP0000283426)

Family Verbenaceae 마편초과

120. Callicarpa dichotoma (Lour.) K. Koch 좀작살나무

Examined specimens: Korea. Chungcheongnam-do: Seosan, 1 Nov 1984 (NIBRVP0000283551, NIBRVP0000283552, NIBRVP0000 283553, NIBRVP0000283670). Gangwon-do: Mt. Geumgang, 17 Feb $1990 \dagger$ (NIBRVP0000283326). Jeollanam-do: Mt. Madung, 22 Jun $1988 \dagger($ NIBRVP0000283522)

121. Callicarpa japonica Thunb. 작살나무

Examined specimens: Korea. Without specific locality: 15 Dec 1905 (NIBRVP0000283297, NIBRVP0000283298). Gyeonggi-do: Incheon, Is. Daecheong, 12 Oct 1984 (NIBRVP0000283305, NIBRVP0000283306, NIBRVP0000283700, NIBRVP0000283701). Jeollanam-do: Mt. Duryun, 25 Oct $2004 \dagger$ (NIBRVP0000283462, NIBRVP0000283523)

122. Callicarpa japonica var. luxurians Rehder 왕작살나무

Examined specimens: Korea. Gyeongsangbuk-do: Is. Ulleung, 24 Oct $1983 \dagger$ (NIBRVP0000283689, NIBRVP000028369, NIBRVP 0000283691)

123. Callicarpa mollis Siebold \& Zucc. 새비나무 Examined specimens: Korea. Jeollanam-do: Mt. Duryun, 25 Oct $2004 \dagger$ (NIBRVP0000283299)

124. Clerodendrum trichotomum Thunb. 누리장나무 Examined specimens: Korea. Gangwon-do: Mt. Seorak, 7 Oct 1977 (NIBRVP0000283409). Jeolla-do: Mt. Jiri, 15 Mar $1983 \dagger$ (NIB RVP0000283662, NIBRVP0000283663)

Family Oleaceae 물푸레나무과

125. *Abeliophyllum distichum Nakai 미선나무

Examined specimens: Korea. Without specific locality: No collection date (NIBRVP0000283420); 20 Jun $1951 \dagger$ (NIBRVP000028 3421); 17 Feb $1951 \dagger$ (NIBRVP0000283203, NIBRVP0000283243, NIBRVP0000283244, NIBRVP0000283419)

126. Chionanthus retusus Lindl. \& Paxton 이팝나무

Examined specimens: Korea. Gyeonggi-do: Seoul, Seodaemun, 22 May $1987 \dagger$ (NIBRVP0000283566, NIBRVP0000283586). Jeollanam-do: Haenam, 24 Oct $1983 \dagger$ (NIBRVP0000283486, NIBRVP0000283512, NIBRVP0000283573)

127. *Forsythia koreana (Rehder) Nakai 개나리 Examined specimens: Korea. Gyeonggi-do: Suwon, 11 Feb $1968 \dagger$ (NIBRVP0000283455)

128. *Forsythia ovata Nakai 만리화 Examined specimens: Korea. Without specific locality: No collection date (NIBRVP0000283222). Gangwon-do: 4 Dec $1986 \dagger$ (NIBRVP0000283442); Mt. Geumgang, 1 Jul 1918 (NIBRVP0000283230, NIBRVP0000283441)

129. *Fraxinus chiisanensis Nakai 물들메나무

Examined specimens: Korea. Gangwon-do: Mt. Geumgang, 6 May $1985 \dagger$ (NIBRVP0000283453)

130. Fraxinus mandshurica Rupr. 들메나무

Examined specimens: Korea. Gangwon-do: Mt. Seorak, 17 May $1977 \dagger$ (NIBRVP0000283449, NIBRVP0000283450, NIBRVP000 0283451)

131. Fraxinus rhynchophylla Hance 물푸레나무

Examined specimens: Korea. Without specific locality: 28 Dec 1906† (NIBRVP0000283677); 4 Mar 1982† (NIBRVP0000283448). Gangwon-do: Mt. Seorak, 17 May $1977 \dagger$ (NIBRVP0000283515)

132. *Ligustrum foliosum Nakai 섬쥐똥나무

Examined specimens: Korea. Gyeonggi-do: Seoul, Forest Research Institute, 17 Oct $1977 \dagger$ (NIBRVP0000283229, NIBRVP0000 283459, NIBRVP0000283460)

133. Ligustrum japonicum Thunb. 광나무 Examined specimens: Korea. Jeollanam-do: Is. Daeheuksan, 23 Sep 1985 (NIBRVP0000283558, NIBRVP0000283559)

134. Ligustrum obtusifolium Siebold \& Zucc. 쥐똥나무

Examined specimens: Korea. Gyeonggi-do: Seoul, 21 Sep 1905† (NIBRVP0000283456, NIBRVP0000283457, NIBRVP0000283458); Forest Research Institute, 5 Oct 1977 (NIBRVP0000283446, NIBRVP0000283447, NIBRVP0000283467, NIBRVP0000283468, NIBRVP0000283469)

135. Ligustrum ovalifolium Hassk. 왕쥐똥나무 Examined specimens: Korea. Jeju-do: Samgumburi, 22 Jun $1988 \dagger$ (NIBRVP0000283464) 
Appendix 1. Continued.

136. Ligustrum salicinum Nakai 버들쥐똥나무

Examined specimens: Korea. Gyeonggi-do: Seoul, Forest Research Institute, 5 Oct 1977 (NIBRVP0000283684)

137. *Syringa oblata var. dilatata (Nakai) Rehder 수수꽃다리

Examined specimens: Korea. North Korea. Without specific locality: 1 Jul 2001 (NIBRVP0000283477, NIBRVP0000283487). Gangwon-do: Mt. Geumgang, 23 Feb $1987 \dagger$ (NIBRVP0000283418). Hamgyeongbuk-do: 1 Jul 1917 (NIBRVP0000283488, NIBRVP00 00283417)

138. Syringa patula (Palib.) Nakai 털개회나무

Examined specimens: Korea. Gangwon-do: Mt. Seorak, 13 Aug 1982 † (NIBRVP0000283482)

139. Syringa reticulata var. mandshurica (Maxim.) H. Hara 개회나무 Examined specimens: Korea. Without specific locality: 1 Jul 1917 (NIBRVP0000283223)

140. Syringa wolfii C.K. Schneid. 꽃개회나무

Examined specimens: Korea. Yanggang-do: Gaema Plateau and Mt. Baekdu, 6 May $1985 \dagger$ (NIBRVP0000283436, NIBRVP00002 83479)

141. Syringa wolfii var. hirsuta (C. K. Schneid.) Hatus. 털꽃개회나무

Examined specimens: Korea. Jella-do: Mt. Jiri, 22 Mar 1977 † (NIBRVP0000283228, NIBRVP0000283440, NIBRVP0000283694)

Family Scrophulariaceae 현삼과

142. Paulownia tomentosa (Thunb.) Steud. 참오동나무

Examined specimens: Korea. Gangwon-do: Mt. Seorak, 7 Oct 1977 (NIBRVP0000283225, NIBRVP0000283226)

Family Bignoniaceae 능소화과

143. Catalpa ovata G. Don 개오동

Examined specimens: Korea. Jeollabuk-do: Mt. Moak, 17 May 2002† (NIBRVP0000283473, NIBRVP0000283474, NIBRVP0000 283478)

Family Diervillaceae 병꽃나무과

144. Weigela florida (Bunge) A. DC. 붉은병꽃나무

Examined specimens: Korea. Without specific locality: 21 Jun 1984† (NIBRVP0000283318). Gyeonggi-do: Incheon, Is. Daecheong, 10 Aug $1992 \dagger$ (NIBRVP0000283483). Gangwon-do: Mt. Odae, 16 Jun $1994 \dagger$ (NIBRVP0000283320, NIBRVP0000283327)

145. Weigela praecox (Lemoine) L. H. Bailey 소영도리나무

Examined specimens: Korea. Gyeonggi-do: Incheon, Is. Baengnyeong, 17 Oct 1984 (NIBRVP0000283699)

146. *Weigela subsessilis (Nakai) L. H. Bailey 병꽃나무

Examined specimens: Korea. Gangwon-do: Mt. Gyebang, 8 Oct 1977 (NIBRVP0000283544, NIBRVP0000283545); 22 Mar 1983 $\dagger$ (NIBRVP0000283546). Gyeonggi-do: Mt. Yongmun, 9 Oct 1977 (NIBRVP0000283321, NIBRVP0000283322, NIBRVP000028 3323, NIBRVP0000283210, NIBRVP0000283194, NIBRVP0000283324, NIBRVP0000283325, NIBRVP0000283547). Jeju-do: Mt. Halla, 4 May $1993 \dagger$ (NIBRVP0000283319); 1 Jan 2000 (NIBRVP0000283191, NIBRVP0000283209, NIBRVP0000283485). Jeollanam-do: Jindo, 5 Sep 1985 (NIBRVP0000283195, NIBRVP0000283196)

Family Caprifoliaceae 인동과

147. Lonicera chrysantha Turcz. ex Ledeb. 각시괴불나무 Examined specimens: Korea. Gangwon-do: Mt. Gyebang, 8 Oct 1977 (NIBRVP0000283271, NIBRVP0000283273)

148. Lonicera maackii (Rupr.) Maxim. 괴불나무

Examined specimens: Korea. Gangwon-do: Mt. Gyebang, 8 Oct 1977 (NIBRVP0000283279, NIBRVP0000283280, NIBRVP00002 83281, NIBRVP0000283282, NIBRVP0000283283, NIBRVP0000283284, NIBRVP0000283285, NIBRVP0000283286, NIBRVP 0000283287)

149. *Lonicera subsessilis Rehder 청괴불나무 Examined specimens: Korea. Hamgyeongnam-do: 2 Aug 1917 (NIBRVP0000283602, NIBRVP0000283603, NIBRVP0000283604)

Family Viburnaceae 산분꽃나무과

150. Viburnum carlesii Hemsl. 분꽃나무

Examined specimens: Korea. Chungcheongnam-do: Cheollipo Arboretum, 1 Jan $1977 \dagger$ (NIBRVP0000283609, NIBRVP0000283610), Taean, 2 Oct 1977 (NIBRVP0000283607, NIBRVP0000283608). Jeollanam-do: Mt. Wolchul, 15 Mar 1983† (NIBRVP0000283612)

151. Viburnum dilatatum Thunb. 가막살나무

Examined specimens: Korea. Jeollanam-do: Mt. Mudeung, 13 Oct 1977 (NIBRVP0000283413, NIBRVP0000283414, NIBRVP00 00283415, NIBRVP0000283416, NIBRVP0000283605, NIBRVP0000283606). Gyeonggi-do: Incheon, Is. Baengnyeong,19 Oct 1984 (NIBRVP0000283411, NIBRVP0000283412)

152. Viburnum erosum Thunb. 덜꿩나무

Examined specimens: Korea. Without specific locality: no collection date (NIBRVP0000283197). Chungcheongnam-do: Taean, 2 Oct 1977 (NIBRVP0000283611). Gyeonggi-do: Incheon, Is. Daecheong Satandong, 13 Oct 1984 (NIBRVP0000283614); Is. Baengnyeong, 19 Oct 1984 (NIBRVP0000283615, NIBRVP0000283616, NIBRVP0000283617). Jeollanam-do: Mt. Wolchul, 15 Mar $1983 \dagger($ NIBRVP0000283613)

153. Viburnum opulus var. calvescens (Rehder) H. Hara 백당나무

Examined specimens: Korea. Jeollabuk-do: Buan, 9 Oct 1985 (NIBRVP0000283542, NIBRVP0000283567)

Family Smilacaceae 청미래덩굴과

154. Smilax sieboldii Miq. 청가시덩굴

Examined specimens: Korea. Without specific locality: 6 Jul $2000 \dagger$ (NIBRVP0000283310) 